Participants 510 elite- and sub-elite indoor sport athletes with at least one previous LAS injury within the preceding 24 months were enrolled; 480 completed the trial.

Intervention Spraino ${ }^{\circledR}$; a low-friction patch applied to the lateral side of the shoe.

Main Outcome Measurements The trial was explorative with evenly-valued outcome measures related to incidence and severity of self-reported LAS injuries, pain in the ankle, fear of injury and intervention-related adverse events.

Results A total of 151 LAS injuries were reported within the trial period, of which 96 were categorized as non-contact injuries. A total of 50 injuries were severe. All metrics favoured Spraino ${ }^{\circledR}$ with computed incidence rate ratios of $0.87(95 \%$ CI, 0.62-1.23) for any LAS injury, 0.64 (95\% CI, 0.42-0.97) for non-contact LAS injuries, and 0.41 (95\% CI, 0.19-0.89) for severe non-contact LAS injuries. The relative time-loss for the total number of injuries was 0.65 (95\% CI, 0.45-0.93). Fear-of-injury and ankle pain was also lower in the Spraino ${ }^{\circledR}$ group. Six participants reported minor harms due to slipping on the floor because of Spraino ${ }^{\circledR}$.

Conclusions Spraino ${ }^{\circledR}$ was found to be effective and safe when used to prevent LAS injuries in indoor sports. Findings should be replicated in a confirmatory RCT.

Trial registration ClinicalTrials. gov: NCT03311490

Funding Innovation Fund Denmark (7038-00087A)

\section{FLOORBALL PARTICIPATION, INJURY PREVENTION EXPECTATIONS, INJURY RISK PERCEPTIONS AND HEALTH PROBLEMS IN SWEDISH YOUTH PLAYERS AT THE START OF A SEASON}

1,2,3,4 Nirmala Perera, ${ }^{1}$ Ida Åkerlund, ${ }^{1}$ Martin Hägglund. ${ }^{1}$ Sport Without Injury ProgrammE (SWIPE), Division of Physiotherapy, Department of Medical and Health Sciences, Linköping University, Linköping, Sweden; ${ }^{2}$ Nuffield Department of Orthopaedics, Rheumatology, and Musculoskeletal Sciences, University of Oxford, Oxford, UK; ${ }^{3}$ Centre for Sport, Exercise and Osteoarthritis Research Versus Arthritis, Oxford, UK; ${ }^{4}$ School of Allied Health, College of Science, Health and Engineering, Latrobe University, Bundoora, Australia

\subsection{6/bjsports-2021-IOC.18}

Background Floorball is a popular sport among Scandinavian youth. However, insufficient data hinders the development of focused injury prevention strategies in floorball.

Objective Describe the motivations for floorball participation as well as injury prevention expectations, injury risk perceptions and the prevalence of health problems in youth players at the beginning of the floorball season.

Design Cross-sectional survey at baseline (2017-2018 season). Setting Swedish youth floorball.

Patients (or Participants) 471 (140 female, 331 male) players.

Main Outcome Measurements Floorball participation, injury prevention/risk perceptions, health problems

Results Female and male players were on average 13.7 $( \pm 1.5)$ and $13.3( \pm 1.0)$ years old, and had played floorball for $4.9( \pm 2.3)$ years. Most (51\% female vs $55 \%$ male) players trained/played floorball 3 times/week; a majority $(69 \%$ female vs $76 \%$ of male) thought their training volume was high. Fractures (84\% female, $90 \%$ male) and eye injuries (90\% female, $83 \%$ male) were perceived to be most severe. $93 \%$ believed sports injuries could be prevented, however, $74 \%$ thought they would not get injured. $85 \%$ (88\% male vs $78 \%$ female) of the players always used protective eyewear.

Females felt more stress (median $=4$, IQR 2-6) than males (median=2, IQR $0-4, \mathrm{P}=0.000$ ), but reported better well-being (female median=3, IQR $1-5$ ) vs (male median $=2$, IQR $0-3, P=0.000)$. No difference in sleep between females (median=3, IQR 1-5) and males, (median=3, IQR 0-3, n. s.) was observed. $33 \%$ (38\% female vs $30 \%$ male) youth players were unable to fully participate in floorball due to health problems at the start of the season, and $65 \%$ of these were injuries. $28 \% \quad(32 \%$ female vs $26 \%$ male) reported pain.

Conclusions This study provides insight into youth players' health status leading into the season; one in three reported a health problem and if these are untreated, there is a potential for more severe and long-term adverse health consequences. Safe sports programmes should be a priority.

\section{WE HAVE THE INJURY PREVENTION PROGRAMME, BUT HOW WELL DO YOUTH USE IT?}

1,2,3,4 Nirmala Perera, ${ }^{1}$ Martin Hägglund. 'Sport Without Injury ProgrammE (SWIPE), Division of Physiotherapy, Department of Medical and Health Sciences, Linköping University, Linköping, Sweden; ${ }^{2}$ Nuffield Department of Orthopaedics, Rheumatology, and Musculoskeletal Sciences, University of Oxford, Oxford, UK; ${ }^{3}$ Centre for Sport, Exercise and Osteoarthritis Research Versus Arthritis, Oxford, UK; ${ }^{4}$ School of Allied Health, College of Science, Health and Engineering, Latrobe University, Bundoora, Australia

\subsection{6/bjsports-2021-IOC.19}

Background Over the past two decades, sports medicine research has developed innovative and proven interventions for injury prevention in athletes. Intervention effectiveness of any injury prevention exercise programme (IPEP) is influenced by both utilisation and exercise fidelity, but this has rarely been evaluated in previous randomised controlled trials (RCT). Objective To describe the exercise fidelity and utilisation fidelity of the Knee Control IPEP in youth floorball alongside an intervention RCT.

Design Observation study, 26-week season.

Setting Swedish youth floorball.

Patients (or Participants) 20 teams (8 female, 12 males) aged 12-17 years.

Interventions (or Assessment of Risk Factors) Knee Control IPEP.

Main Outcome Measurements Exercise fidelity and program utilisation fidelity.

Results Of the 535 individual Knee Control exercises observed, 76\% were performed by males; and 58\% exercises were performed correctly. Exercise fidelity was greater in females $(71 \%$ vs $54 \%, \mathrm{P}=0.001)$. No difference in exercise fidelity during the first (57\%) and second (59\%) half of the season. The full Knee Control IPEP (7 exercises x 3 sets) was completed as prescribed in only four out of 31 team training sessions observed. Utilisation fidelity did not differ between sexes and the average number of completed exercises performed was $11( \pm 5)$. Males performed more exercises with a higher level of difficulty $(n=247,93$ and 59 for levels A, B and $C+D$, respectively) compared to females $(n=88,26$, and 7, $\mathrm{P}=0.021$ ). $33 \%$ of the coaches perceived that they had good knowledge about injury prevention, only 33\% believed regular IPEP use could decrease injury risk. 
Conclusions The exercise fidelity was low with only three out of five exercises observed performed according to instructions. Only half of the total programme exercises were being executed. Only a third of coaches believed IPEP can reduce injury risk; thus current delivery strategies might be insufficient for translating evidence to this key-stakeholder group. Future work is needed to inform evidence-based strategies to better support the implementation of IPEPs in sport settings.

\section{THE EFFECT OF A WORKSHOP ON COACHES' ADOPTION AND ADHERENCE TO THE ACTIVATE INJURY PREVENTION EXERCISE PROGRAMME}

${ }^{1}$ Craig Barden, ${ }^{1,2}$ Carly McKay, ${ }^{1,3}$ Keith Stokes. 'Department for Health, University of Bath, Bath, BA2 7AY, UK., Bath, UK; ${ }^{2}$ Centre for Motivation and Health Behaviour Change, University of Bath, Bath, BA2 7AY, UK., Bath, UK; ${ }^{3}$ Rugby Football Union, Twickenham, TW2 7BA, UK, London, UK

\subsection{6/bjsports-2021-IOC.20}

Background Educational workshops have been employed in football to positively influence coach perceptions towards injury risk and prevention, and to improve adherence to neuromuscular training programmes. Similar implementation strategies have been used in rugby but their effect on behaviour is unknown.

Objective To assess coaches' perceptions towards injury risk and prevention, and to determine the effect of workshop attendance on their uptake of Activate, a rugby-specific injury prevention exercise programme.

Design Prospective cohort study.

Setting English school rugby union (under-12 to under-18).

Participants School rugby coaches and support staff $(n=54)$.

Interventions All coaches completed a baseline questionnaire investigating their perceptions towards injury risk and prevention. Coaches were invited to attend a pre-season Activate workshop.

Main outcome measures Participants were grouped by workshop attendance (yes/no). Perceptions towards injury risk and prevention were compared at baseline, prior to workshop attendance (Mann-Whitney-U test). Coaches self-reported adoption (programme use; chi-square test) and adherence (sessions completed per week; Mann-Whitney-U test) at postseason.

Results Thirty-three of 54 coaches attended a pre-season workshop. Prior to the workshop, $72 \%$ of coaches were aware of Activate. At baseline, attendees were significantly more likely to believe that exercises shown to prevent injuries should be included in rugby training $(\mathrm{z}=-2.03, \mathrm{p}=0.04)$ and completing a specific warm-up prior to every match would improve players' physical characteristics $(z=-2.13, p=0.03)$. Coaches attending a workshop were significantly more likely to adopt Activate $\left(\chi^{2}=5.05, \mathrm{p}=0.02\right)$, with $17 \%$ greater adherence $(\geq 2$ times per week; $\mathrm{z}=-2.20, \mathrm{p}=0.03)$.

Conclusions Coaches with stronger baseline perceptions regarding injury prevention were more likely to attend a workshop and had significantly greater programme adoption and adherence. If coach perceptions are a determinant of workshop attendance, and subsequently programme uptake, researchers and stakeholders should focus upon strategies to influence coach perceptions towards injury risk and prevention.

\section{CAN A MASSIVE OPEN ONLINE COURSE (MOOC) INFORM CONCUSSION PREVENTION KNOWLEDGE TRANSLATION?}

${ }^{1}$ Stephanie Adams, ${ }^{2}$ Pierre Fremont, ${ }^{3}$ Jennifer Lock, $4,5,6$ Keith 0 Yeates, $1,8,5,9,6,7$ Carolyn Emery, ${ }^{10}$ Leslie Reid, ${ }^{11}$ Penny Werthner, ${ }^{1,4,5,6,12,13}$ Kathryn Schneider. ${ }^{1}$ Sport Injury Prevention Research Centre, Faculty of Kinesiology, University of Calgary, Calgary, Canada; '2Department of Rehabilitation, Faculty of Medicine, Université Laval, Québec, Canada; ${ }^{3}$ Werklund School of Education, University of Calgary, Calgary, Canada; ${ }^{4}$ Department of Psychology, Faculty of Arts, University of Calgary, Calgary, Canada; ${ }^{5}$ Hotchkiss Brain Institute, Cumming School of Medicine, University of Calgary, Calgary, Canada; 'Alberta Children's Hospital Research Institute, Cumming School of Medicine, University of Calgary, Calgary, Canada; ${ }^{7}$ Community Health Sciences, Cumming School of Medicine, University of Calgary, Calgary, Canada; ${ }^{8}$ Department of Pediatrics, Cumming School of Medicine, University of Calgary, Calgary, Canada; ${ }^{9} O^{\prime}$ Brien Institute for Public Health, Cumming School of Medicine, University of Calgary, Calgary, Canada; ${ }^{10}$ Faculty of Science, University of Calgary, Calgary, Canada; ${ }^{11}$ Faculty of Kinesiology, University of Calgary, Calgary, Canada; ${ }^{12}$ Acute Sport Concussion Clinic, Sport Medicine Centre, University of Calgary, Calgary, Canada; ${ }^{13}$ Evidence Sport and Spinal Therapy, Calgary, Canada

\subsection{6/bjsports-2021-IOC.21}

Background To reduce the burden of concussion, effective prevention strategies are required. These should: 1) focus on primary prevention in addition to secondary prevention of recurrence and 2) promote global engagement. A Massive Open Online Course (MOOC) on concussion allows stakeholders to interact with experts while learning about the latest evidence and resources on concussion prevention, and may contribute to enhancing awareness and knowledge of prevention.

Objective To evaluate the feasibility and effectiveness of a MOOC, which includes a focus on concussion prevention, as a global knowledge translation (KT) strategy with multiple stakeholders.

Design De-identified learning analytics were used to assess participation, completion rates, and knowledge gains.

Setting Worldwide web.

Participants Data from 12751 self-registered learners (e.g., health professionals, coaches, sports organisations, athletes, parents, teachers, school administrators) was analysed over 2 iterations of the course.

Intervention The MOOC was available for 9 weeks and included six modules: introduction to concussion, primary prevention, detection (secondary prevention), management, treatment (tertiary prevention) and implementation/revision of protocols. Each module featured interactive learning content/ materials and a reflective process supporting KT and evaluations (for more: https://www.ucalgary.ca/knes/online-concussioncourse).

Main outcome measurements Number of registered learners accessing and completing each module; post-module exam scores; pre-test and post-course exam scores.

Results Of 12751 people who registered, 4195 (33\%) successfully completed all evaluations. The modules were highly accessed by participants [introduction $=7212$ (57\%), prevention=5906 (46\%), detection=5149 (40\%), management $=4716$ $(37 \%)$, treatment $=4468(35 \%)$, protocols $=4241(33 \%)]$. For the first iteration, the prevention module had the highest mean post-module exam score 92.06\% [95\% CI; 91.7692.36)] and there was a $12 \%$ increase in overall knowledge between pre- and post-course exam scores (mean score increased from $67 \%$ to $79 \%$ ).

Conclusions The sustained engagement of the participants suggests that a MOOC on concussion may be an effective 